\title{
Arteriovenous subclavian artery fistula with aneurysm secondary to firearm trauma: Case report
}

\author{
Miguel A. Sierra-Juárez ${ }^{1 *}$, Christian Cruz-Romero ${ }^{2}$, Patricia Rodríguez-Nava ${ }^{3}$ and Ernesto. J. Dena-Espinoza ${ }^{4}$ \\ ${ }^{1}$ Service of Vascular Surgery; ${ }^{2}$ Service of General Surgery; ${ }^{3}$ Department of Computed tomography; ${ }^{4}$ Service of Radiology and Imaging, Hospital \\ General de México "Dr. Eduardo Liceaga," Mexico City, Mexico
}

\begin{abstract}
We present the case of a 25-year-old male patient with a history of gunshot wound in the chest 3 years before, who developed symptoms in the arm for 1 year. The patient was assessed by the outpatient clinic, presenting left subclavian murmur, and development of venous network. Three-dimensional rotational angiography revealed left subclavian arteriovenous fistula with aneurysmal dilatation. Patient was operated and received a polytetrafluorethylene graft with excellent evolution. The case is presented due to the complexity of a late vascular injury in a region that is difficult to approach with very high morbidity and mortality rates.
\end{abstract}

Key words: Vascular trauma. Arteriovenous fistula. Subclavian artery.

\section{Introduction}

Vascular injury (especially in a major artery) is a common cause of disability and mortality. According to one report, it accounts for $20-26 \%$ of trauma-induced death. Vascular repair and remodeling are determined by the type and site of injury, concomitant injuries, presence of collateral circulation in the extremities, available techniques, and material'.

The delay in diagnosis and treatment can be catastrophic, leading to aneurysms, arteriovenous fistulas (AVF), and/or gangrene, making it even more difficult to repair blood vessels and increasing the risk of amputation and physical disability. Therefore, "rapid diagnosis and treatment" (within 6-12 h) is the fundamental principle when treating vascular trauma of an extremity, which significantly improves survival ${ }^{2}$.

AVF represents a communication between the high-pressure system and low-pressure system. As
AVF has less blood resistance than the capillary bed, some of the blood from the artery proximal to the fistula tends to pass into the vein. In addition, there is a decrease in peripheral resistance at the site of the fistula and an increase in the gradient of the proximal artery, which will depend on the arterial caliber, the size of the fistula and the resistance of the venous drainage ${ }^{3}$.

Medical history and physical examination are sufficient for a diagnosis of traumatic AVF in almost all cases. Physical examination findings are usually typical and involve a continuous palpable murmur on auscultation and may include signs of chronic venous stasis such as ulceration, pigmentation, edema, and varicose veins sometimes with increased skin temperature. Depending on the size and location of the fistula, congestive heart failure may also develop. The main systemic effects include increased cardiac output, total blood volume, venous pressure, and increased heart rate along with cardiomegaly ${ }^{4}$.

\section{Correspondence:}

*Miguel A. Sierra-Juárez

E-mail: sierrajma@ @otmail.com NC-ND license (http://creativecommons.org/licenses/by-nc-nd/4.0/).
Available online: 22-01-2021

Rev Med Hosp Gen Mex. 2021;84(1):28-31 www.hospitalgeneral.mx 


\section{Case study}

A male patient, with a previous firearm injury in the anterior aspect of the chest (left clavicle region), consulted with a private doctor 3 years ago, because of an arm edema and pain. The doctor decided to refer the patient to vascular surgery. On admission to the outpatient clinic, asymmetry of the shoulder and left arm was observed due to the presence of increased volume, abundant collateral venous network, $3 \mathrm{~mm}$ cephalic vein with pulsed flow, and increased temperature in the clavicular region was felt with continuous murmur on auscultation with absent left ulnar and radial pulse. Angiography was requested where it was possible to view vascular injury of the left subclavian artery with AVF that leads to an increase in venous pressure with multiple collateral venous networks in the elbow and arm, aneurysm sac of the subclavian artery with arteriomegaly, and tortuous vessels of the arm (Fig. 1).

The patient was scheduled for an open surgery in the operating room. He was placed in a supine position; the head was turned toward the right shoulder with vertical roll placement between the scapulae (Fig. 2). A $10-\mathrm{cm}$ long incision was made parallel to the clavicle (trans-clavicular), as well as dissection of the sternocleidomastoid and omohyoid muscle at the clavicular head level. The clavicle was exposed and the fat pad was separated with Gigli saw, with exposure of anterior scalene muscle and external jugular vein. The phrenic nerve was isolated with disinsertion of the anterior scalene muscle to achieve exposure of the subclavian artery. During the layer-by-layer dissection, multiple high-flow venous networks could be seen, which were exposed and ligated. Thrill was palpated at the exposure level of the control proximal subclavian artery and veins, as well as distal control with vascular ligation (Surg-I-Loop). Because of fibrosis and tissue adhesion, no thoracic duct was identified. Arterial aneurysm sac was opened and the fistula hole was identified with the subclavian vein, which was stitched with 5-0 continuous prolene suture. A ringed polytetrafluorethylene (PTFE) vascular graft straight 7-mm was implanted with 6-0 prolene suture (Fig. 3). Surgical time corresponded to hours with general anesthesia with tracheal intubation.

Patient presented with a pneumothorax, which was managed with tube thoracostomy. The patient evolved toward improvement with a three-phase radial and ulnar pulse of good intensity in the left arm, absence of murmur and collapse of the venous networks, as well as scarce chylous fluid leakage through the endopleural

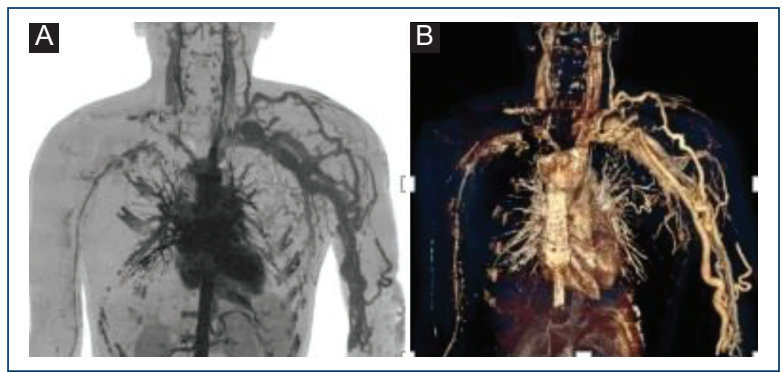

Figure 1. A-B: three-dimensional rotational angiography. Arteriovenous fistula of the left subclavian artery is observed with arterial aneurysm and venous hypertension (arteriomegaly of the arm vessels).

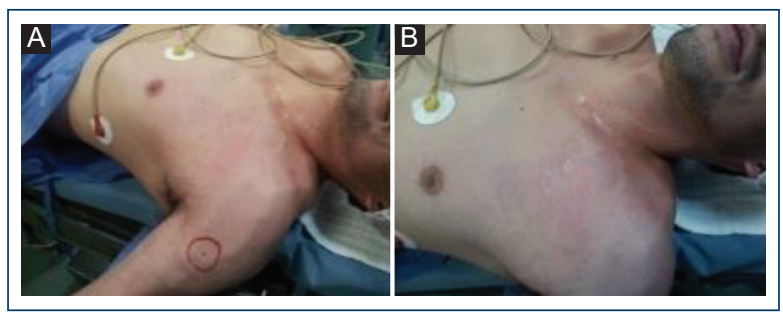

Figure 2. A-B: increased volume of shoulder and arm with multiple dilated venous networks. Continuous murmur is auscultated in left subclavian artery with absence of radial and ulnar pulse.

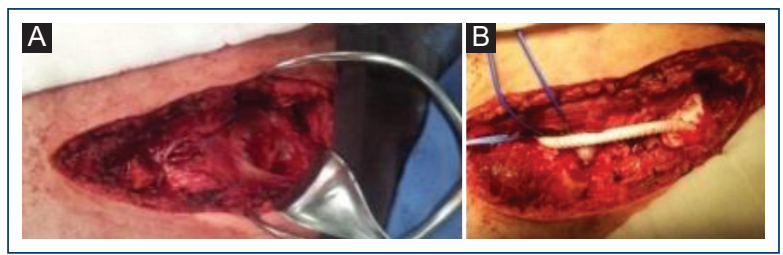

Figure 3. A: exposure of left subclavian artery of appreciable aneurysm sac after removal of clavicle, B: ringed polytetrafluorethylene vascular graft straight.

tube, which was removed on the $5^{\text {th }}$ day. The patient was discharged due to improvement, under control by the outpatient clinic. The patient remained in hospital for 7 days for the procedure and the follow-up consultation took place 2 months later, where he was discharged due to full recovery (Fig. 4).

\section{Discussion}

The overall incidence of subclavian artery and axillary nerve injuries is often due to penetrating trauma, representing $<9 \%$ of all vascular injuries. Subclavian 


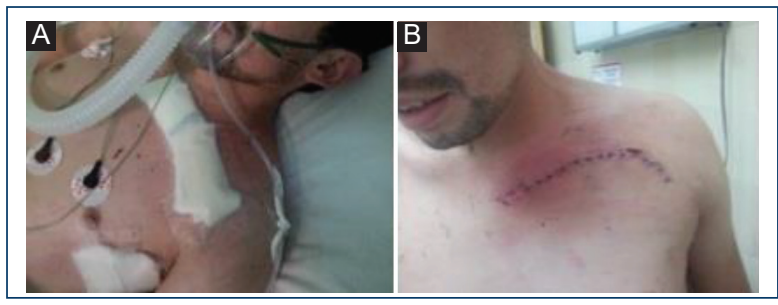

Figure 4. A-B: surgical result of left subclavian arteriovenous fistula closure with absence of venous hypertension with normal radial and ulnar pulse.

artery injuries are associated with high mortality rates and remain fatal, despite advances in modern medicine. Management is particularly challenging due to the anatomical location with the third portion of the subclavian artery located within the chest cavity, which makes exposure difficult, because of the numerous neurovascular structures. Thus, the risk of collateral damage during vascular repair or associated injuries is increased. The subclavian muscle, clavicle, first rib, and deep cervical fascia, as well as the acromioclavicular ligaments and axillary fascia protect the subclavian artery. In addition to difficult exposure, open vascular repairs are associated with longer surgical time, more hospitalization days and higher incidence of post-operative complications ${ }^{5}$. We presented the case of a firearm injury in the subclavian artery that turned into a high-output fistula with venous hypertension and aneurysm sac. This type of injury is very complex to repair due to the difficulties in accessing the anatomical structures. Sometimes, it is necessary to remove the clavicle to improve exposure and be able to have better vascular control of the healthy proximal and distal ends of the vascular network.

Traditional open surgery with direct vascular repair has been the mainstay of surgical management using many approaches, including midline sternotomy, anterolateral thoracotomy involving supra and infra-clavicular and trans-clavicular approaches. Chest wall rigidity and disruption of tissue layers due to trauma can make it difficult to control bleeding in open surgery. As for the access to proximal subclavian and axillary artery injuries, the mortality rate has been reported as high as $30 \%{ }^{6}$. The decision regarding the best approach is fundamental. The trans-clavicular approach was chosen because the injury was located in the medial third of the clavicle, making a thoracic approach difficult. This approach would allow us, if necessary, to extend the incision toward the axillary region.
In some cases, even extending the incision toward the thorax. Limb loss rates of $2.9 \%$ have been reported in patients with subclavian and axillary vessel injuries. Combined arterial and venous injuries are rare but associated with a mortality rate of up to $61 \%$ in patients who are still alive on hospital admission? ${ }^{7}$ Injuries to the subclavian artery and vein with the consequent formation of a fistula prevented rupture of the vessel with hemorrhage to the chest and hemorrhagic shock, which meant that the patient remained asymptomatic for several years but developed high venous hypertension with early signs of fluid overload.

Ostovan presents the endovascular repair of traumatic subclavian-axillary artery dissection in four patients with limb ischemia successfully managed by the endovascular approach with an uncoated self-expanding metal stent. This led to excellent immediate results with limb recovery and without further complications ${ }^{8}$. Endovascular therapy to repair subclavian injuries is a viable option but has some contraindications: large lesions, absence of proximal neck anchorage, discrepancy between proximal and distal end vessels, impossibility of vessel catheterization, and injuries that require exploratory surgery, hematoma with compression symptoms, and infected injuries. The deformity that occurred in the vessels due to the high output fistula caused the artery to be deformed (arteriomegaly) which made the anchoring of the stent impossible because of the discrepancy in the lumen of the vessels.

A National Trauma Data Bank $®$ review searched for all traumatic axillosubclavian artery injuries (ASAl) over 2 years 2010-2012 and retrospectively for a single center for consecutive patients with ASAI between January 2010 and August 2014. The review included 511,286 patients with $520 \mathrm{SAl}$, with an incidence of $0.1 \%$. Endovascular therapy was used in 76 patients (14.7\%) versus open repair in 280 patients $(53.8 \%)$, resulting in a series in which $30 \%$ of the stents were occluded within 132 days. The published literature is difficult to interpret due to publication bias favoring successful outcomes. Poor follow-up prevents a management strategy of interval surveillance of stents, with intervention reserved only for those with stenosis in the stent in an effort to help primary patency of stenting ${ }^{9}$. One of the great advantages of open surgery compared to stenting, despite its risks, is the presence of long-lasting patency. We used a PTFE graft, as we did not have a suitable venous graft in terms of diameter.

Maués reports the case of a patient with a firearm injury with a computed tomography scan showing a subclavian AVF, which was repaired with an $8 \times 100 \mathrm{~mm}$ coated stent 
with good evolution and closure of the fistula. Short- and medium-term results have revealed low occlusion and IntraStent restenosis rates. Even in cases where occlusion occurs, there is no impediment to revascularization when necessary. The durability of endovascular prosthesis has not been fully established yet and there are no long-term follow-up reports in such cases. For this reason, there is no consensus on the routine use of endovascular therapy in subclavian artery injuries ${ }^{10}$.

Re-interventions for stent occlusion and stenosis can increase patient care costs. One of the most dangerous risks is that the patient will develop ischemia and that further complicates the approach as it requires removal of the stent which may present tissue adhesion. The patient was monitored by the outpatient clinic and his arm mobility and pulses were normal, with absence of pain in the arm.

\section{Conclusions}

Subclavian artery injuries are very complex to repair and can be life threatening. When accompanied by a fistula and aneurysm, they are often fatal.

Suspected subclavian artery injury due to penetrating gunshot wounds requires detailed vascular exploration and close monitoring. Extension studies help confirm the diagnosis and allow these injuries to be treated early without deformity of the vessels with endovascular therapy with coated stent or a less technically complex open surgical approach.

\section{Conflicts of interest}

The authors declare that they have no conflicts of interest.

\section{Ethical disclosures}

Protection of human and animal subjects. The authors declare that the procedures followed were in accordance with the regulations of the relevant clinical research ethics committee and with those of the Code of Ethics of the World Medical Association (Declaration of Helsinki).

Confidentiality of data. The authors declare that no patient data appear in this article.

Right to privacy and informed consent. The authors declare that no patient data appear in this article.

\section{References}

1. Zhui L, Liang Z, Kaizhen W. Characteristics and treatment of vascular injures: a review of 387 cases at a Chinese center. Int J Clin Exp Med. 2014;7:4710-9.

2. Xu Y, Wenjing, Wang A, Meng $H$, Wang $Y$, Liu S, Li R, et al. Diagnosis and treatment of traumatic vascular injury of lims in military and emergency medicine: a systematic review. Medicine. 2019;98:e15406.

3. Varela-Jiménez I, Gutiérrez-Arias E. Fistula arterio venosas traumáticas. Salus. 2017;21:26-9.

4. Mazlum S, Cihan Y, Eyup MK, Mert FT. Management of traumatic arteriovenous fistulas: a tertiary academic center experience. Ulus Trauma Acel Cerrahi Derg. 2018;24:234-8.

5. Eikbuli A, Shaikh S, McKenney M, Boneva D. Successful management with endovascular stent graft repair following gunshot wound to the subclavian artery: case report and literature review. Int J Surg Case Rep. 2019;64:75-79.

6. Waller CJ, Cogbill TH, Kallies KJ, Ramirez LD, Cardenas JM, Todd SR, et al. Contemporary management of subclavian and axillar artery injuries a Western Trauma association multicenter review. J Trauma Acute Care Surg. 2017;83:1023-31.

7. Grigorian A, Wilson SE, de Virgilio C, Kabutey NK, Fujitani RM, Gabriel V, et al. Humerus fracture and combined venous injury increases limb loss in axillary or subclavian artery injury. Vascular. 2019;27:252-9.

8. Ostovan MA, Kojuri J, Dehghani P. Endovascular repair of the traumatic dissection of the subclavian axillary artery: report of four cases. J Tehran Heart Cent. 2017;12:88-91.

9. Chopra A, Modrall JG, Knowles M, Phelan HA, Valentine RJ, Chung J. Uncertain patency of covered stents placed fr traumatic axillosubclavian artery injury. J Am Coll Surg. 2016;223:174-83.

10. Maués JJ, Hauter HL. Endovascular treatment of traumatic subclavian arteriovenous fistula: case report. J Vasc Bras. 2018;17:248-51. 